\section{(C) OPEN ACCESS}

\title{
Efficacy of intensive multidisciplinary rehabilitation in Parkinson's disease: a randomised controlled study
}

\author{
Davide Ferrazzoli, ${ }^{1}$ Paola Ortelli, ${ }^{1}$ Ilaria Zivi, ${ }^{1}$ Veronica Cian, ${ }^{1}$ Elisa Urso, ${ }^{1}$ \\ Maria Felice Ghilardi, ${ }^{2}$ Roberto Maestri, ${ }^{3}$ Giuseppe Frazzitta $^{1}$
}

'Department of Parkinson's Disease, Movement Disorders and Brain Injury Rehabilitation, 'Moriggia-Pelascini' Hospital, Gravedona ed Uniti, Italy ${ }^{2}$ Department of Physiology, Pharmacology and Neuroscience, CUNY Medical School, New York, New York, USA

${ }^{3}$ Department of Biomedical Engineering, Istituti Clinici Scientifici Maugeri Spa Società Benefit, IRCCS, Montescano, Italy

\section{Correspondence to Dr Davide Ferrazzoli,} Department of Parkinson's disease, Movement Disorders and Brain Injury Rehabilitation, 'Moriggia-Pelascini' Hospital, Gravedona ed Uniti 22015 Italy; davideferrazzoli@gmail. com

$\mathrm{PO}, \mathrm{RM}$ and GF contributed equally.

Received 14 May 2017 Revised 21 November 2017 Accepted 11 December 2017 Published Online First 10 January 2018

\section{SLinked}

- http://dx.doi.org/10.1136/ jnnp-2017-317812

Check for updates

To cite: Ferrazzoli D, Ortelli $P$, Zivi I, et al. J Neurol Neurosurg Psychiatry 2018;89:828-835.

\section{ABSTRACT}

Objective To evaluate whether a 4-week

multidisciplinary, aerobic, motor-cognitive and intensive rehabilitation treatment (MIRT) improves the quality of life $(\mathrm{Q} o \mathrm{~L})$ of patients with Parkinson's disease (PD), in the short-term and long-term period.

Methods This is a prospective, parallel-group, singlecentre, single-blind, randomised clinical trial (ClinicalTrials.gov NCT02756676). 186 patients with $\mathrm{PD}$, assigned to experimental group, underwent MIRT; conversely, 48 patients, assigned to control group did not receive rehabilitation. Parkinson's Disease Questionnaire-39 was assessed 2 (T0), 10 (T1) and 18 (T2, only experimental group) weeks after the enrolment. We compared T1 versus T0 scores within subjects and delta scores (T1-T0) between subjects. To investigate the long-term effects, we compared T2 and T0 scores in the experimental group.

Results At T0, no between-group differences in the Global Index Score (GBI) were observed (experimental group: 43.6 \pm 21.4 , controls: 41.6 $\pm 22.9, \mathrm{P}=0.50$ ). At $\mathrm{T} 1$, we did not find significant changes in controls (delta score: $1.2 \pm 9.9, P=0.23$ ), and we found an improvement in $\mathrm{GBI}$ in the experimental group (delta score: $-8.3 \pm 18.0, P<0.0001)$, significant also between subjects $(P<0.0001)$. Comparing T2 versus T0 in the experimental group, the $\mathrm{GBI}$ maintained a significant improvement (delta score: $-4.8 \pm 17.5, \mathrm{P}<0.0001$ ).

Conclusions A rehabilitation treatment such as MIRT could improve QoL in patients with PD in the short-term and long-term period. Even though the single-blind design and the possible role of the placebo effect on the conclusive results must be considered as limitations of this study, the improvement in outcome measure, also maintained after a 3-month follow-up period, suggests the effectiveness of MIRT on the QoL.

Clinical trial registration NCT02756676: Pre-results.

\section{INTRODUCTION}

Quality of life (QoL) is the sense of well-being perceived by people. It represents an important index of the quality of healthcare, ${ }^{12}$ and it is particularly relevant in chronic diseases, such as Parkinson's disease (PD).

$\mathrm{PD}$ is a progressive neurological disorder characterised by motor and non-motor symptoms such as rigidity, bradykinesia, resting tremor, autonomic and cognitive dysfunctions, sleep disorders, and sensory disturbances. The combination of these symptoms reduces patients' QoL, affecting their social and emotional well-being. ${ }^{3}$ In early PD, dopaminergic drugs may help to improve the self-perceived QoL by acting on the predominant motor symptoms. ${ }^{45}$ In the advanced stages of the disease, non-dopaminergic symptoms prevail and the use of the same drugs is less effective. In addition, in this phase the higher levodopa dose $(>400 \mathrm{mg} / \text { day })^{6}$ and the side effects of its long-term use (dyskinesias, dystonia, 'wearing off', dopamine dysregulation syndrome) have a further detrimental impact on the health-related QoL. 7

Recently, rehabilitation has been proposed as a complementary and effective treatment for the management of PD. ${ }^{8-12}$ Its positive effect is documented mainly on postural and gait dysfunctions, well-known for their drug resistance. ${ }^{13-16}$ Exercise seems to act in PD by promoting the so-called 'activity-dependent neuroplasticity, ${ }^{17-19}$ through the intensity, specificity, difficulty and complexity of its activities, ${ }^{17-19}$ and by reducing the need for a progressive rise in dopaminergic therapy. ${ }^{11} 12$ To date, only few studies evaluated the effect of rehabilitation on QoL of patients with PD. As first, Herman et $a l^{20}$ found that a progressive and intensive 6-week treadmill training programme improved gait and QoL in patients with PD. Differently, Paker et $a l^{21}$ showed an improvement of QoL after robotic treadmill training in a group of outpatients with PD. More recently, Morberg et al ${ }^{22}$ proved how a 32-week personalised high-intensity physical training enhanced the emotional well-being and bodily discomfort items of the Parkinson's Disease Questionnaire-39 (PDQ-39) ${ }^{23}$ beyond the motor and non-motor symptoms of PD. ${ }^{22}$ However, the cited studies were limited by the lack of randomisation, the small number of participants and the absence of a multidisciplinary approach, which is considered as beneficial factor in the management of PD. ${ }^{24-26}$ Indeed, even though positive and sustained effects of multidisciplinary interventions in improving the QoL of people living with PD have been shown, ${ }^{27}$ this evidence still needs to be tested and verified in randomised controlled trials using standardised outcome measures, adequate samples and longer follow-up periods. ${ }^{27}$ Nonetheless, the effect of a multidisciplinary care programme on QoL has been already assessed in patients with PD with conflicting results. ${ }^{28} 29$

The aim of the present study is to evaluate whether a multidisciplinary, aerobic, motor-cognitive and intensive rehabilitation treatment (MIRT) ${ }^{111230}$ improves the QoL of patients with $\mathrm{PD}$ in the short-term and long-term period. 


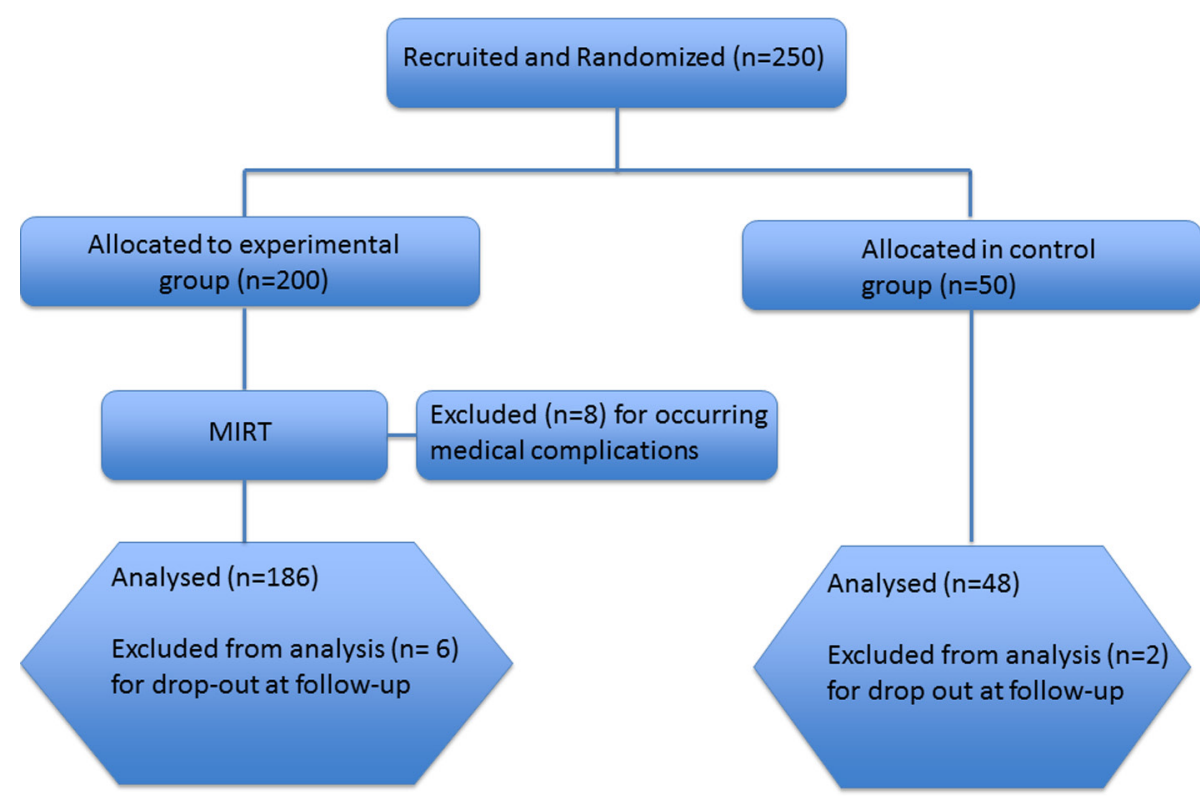

Figure 1 CONSORT diagram. CONSORT, Consolidated Standards of Reporting Trials; MIRT, Multidisciplinary Intensive Rehabilitation Treatment.

\section{MATERIALS AND METHODS}

This is a prospective, parallel-group, single-centre, single-blind randomised clinical trial conducted at the Department of Parkinson's Disease, Movement Disorders and Brain Injury Rehabilitation of the 'Moriggia-Pelascini' Hospital (Gravedona ed Uniti, Italy) between June 2015 and August 2016 (see figure 1).

\section{Participants, randomisation and masking}

Two hundred and fifty patients with idiopathic PD were enrolled from neurologists expert in movement disorders. The following were the eligibility criteria: (1) diagnosis of idiopathic PD according to the UK Brain Bank criteria, ${ }^{31}$ (2) Hoehn and Yahr stages 2-4 and (3) stable pharmacological treatment in the last 6 weeks. The following were the exclusion criteria: (1) any focal brain lesion detected with brain imaging studies (CT or MRI), (2) psychosis (evaluated with Neuropsychiatric Inventory), (3) auditory, visual and/or vestibular dysfunctions, and (4) chronic diseases other than PD with a known impact on QoL. The patients were randomised through a parallel-group design with a 4:1 allocation ratio; therefore, 200 patients were included in the experimental group and 50 served as controls. The allocation ratio was determined by the admission capability of our institute (patients in the control group were put in a waiting list to be admitted later). For the allocation of participants, a list of random numbers was computer-generated. The sequence was concealed to the enrolling neurologists until group assignment. Researchers involved in QoL data collection were blinded to treatment allocation and to the study design, while the other health professionals involved in the study (physiotherapists, nurses, other physicians) were not. Patients enrolled in the experimental group were hospitalised 15 days after the recruitment and underwent a 4-week MIRT. ${ }^{11} 1230$ All patients enrolled in the study did not undergo rehabilitation before their participation in the study, while those enrolled in the control group were required to not perform any kind of rehabilitation during the study period. All patients signed an informed written consent to participation in the study and to the use of their clinical data. This trial was registered on ClinicalTrials.gov website (NCT02756676).

\section{Rehabilitation treatment}

MIRT is a multidisciplinary, aerobic, motor-cognitive, intensive and goal-based rehabilitation treatment specifically designed for patients with PD. ${ }^{11} 1230$ The aim of the treatment is to relearn the dysfunctional movements resulting from the disease through the use of explicit and implicit learning strategies. It consists of a 4-week programme in a hospital setting, composed of four daily rehabilitative sessions for 5 days and 1 hour of physical exercise on the sixth day. The duration of each session, including recovery periods, is about 1 hour.

- The first session consists of a one-to-one treatment with a physical therapist. It comprises cardiovascular warm-up activities, active and passive exercises to improve the joints' range of motion, stretching of the abdominal muscles, strengthening of paravertebral muscles, postural changes, and exercises operating on balance and postural control.

- The second session exploits the use of various devices to improve gait and balance: a stabilometric platform with biofeedback (patients have to follow a pathway on a screen by using a cursor sensitive to their feet movements on the platform), a treadmill plus (treadmill training with visual cues, auditory cues and feedback), ${ }^{32}$ a crossover ${ }^{33}$ and a cycloergometer with feedback. We use a maximum treadmill speed of $3.5 \mathrm{~km} /$ hour; patients are trained with treadmill for no more than 15 min, two times per day.

- The third session consists of occupational therapy aimed to improve the autonomy in everyday activities. The session focuses on hand dexterity, writing and activities of daily living (ADLs). The hand and fingers dexterity training entails exercises aimed at reacquiring the functional use of the most affected hand and the skills in the coordinated activities of both hands. The writing rehabilitation treatment consists of paper-and-pencil exercises and uses visual cues and verbal strategies aimed to enlarge the letters' size and improve the readability. Finally, patients are trained by performing ADLs in the rehabilitation setting, exploiting self-management and cognitive-behavioural strategies.

- The fourth session includes 1 hour of speech therapy. In this field, three possible kinds of intervention are proposed: 
(1) a counselling for patients and caregivers pertinent to a good management of language and swallowing problems; (2) an individual swallowing training, which includes meal monitoring and learning strategies for a correct ingestion of foods and liquids; and (3) a group therapy aimed to treat the hypokinetic dysarthria (breathing exercises to relax and alleviate the pressure of speech; facial exercises to improve the range of facial expressions and mouth motion; exercises to improve vocalisation, articulation and speech prosody).

On the sixth day the patients are trained only with devices for 1 hour.

The rehabilitation programme could also include hydrotherapy in case of severe balance and postural disorders, robotic-assisted walking training for complex gait disorders, virtual reality training and psychoeducational groups with neuropsychologists.

During all the activities, the heart rate reserve is kept between $70 \%$ and $80 \%$.

A weekly team meeting defines the rehabilitation programme for each patient and assesses its benefits during the course of the hospitalisation.

\section{Assessment of outcomes}

Primary outcome measure: PDQ-39

PDQ-39 is a questionnaire specifically designed to assess QoL in patients with PD. ${ }^{23}$ It is composed of 39 items assessing patients' experience of certain events affecting their QoL. The items are grouped into eight issues: mobility, ADLs, emotional well-being, stigma of the disease (stigma), social support, cognition, communication and bodily discomfort. For each item, the patient is asked to indicate how frequent each corresponding event was over the last month (never, occasionally, sometimes, often or always). The final result of the scale includes eight subscores, one Global Index Score and the weighted percentage of the problem's severity. Comparative studies between the PDQ-39 scales and other QoL scales showed a highest sensitivity of PDQ-39 in the evaluation of QoL in patients with PD. ${ }^{23} 34$

The questionnaire was administered to all participants by a trained neuropsychologist at 2 weeks (T0) and 10 weeks (T1) after the enrolment. In the experimental group, T0 corresponds to the hospital admission, while T1 to a 4-week follow-up after discharge. Patients in the experimental group were evaluated with PDQ-39 even at 18 weeks after the enrolment (T2, 12 weeks after discharge) to assess the long-term effect of MIRT on QoL (see figure 2). We choose to set T1 at 4 weeks after the end of treatment (10 weeks after the enrolment), instead of immediately at the end of rehabilitation, in order to avoid bias related to the hospitalisation itself, which could affect patients' judgement. Indeed, this could particularly happen for those PDQ-39 items exploring ADLs and the quality of the relations with the caregiver.

\section{Secondary outcome measures}

Patients in the experimental group were evaluated on the second (pre-MIRT) and last (post-MIRT) day of hospitalisation (see figure 2) with the following clinical, functional and motor scales: Unified Parkinson's Disease Rating Scale (UPDRS), Parkinson's Disease Disability Scale (PDDS), Timed Up and Go Test (TUG) and Berg Balance Scale (BBS). The scores were assessed in the morning, 1 hour after the first dopaminergic dose, by a neurologist and a physiotherapist expert in movement disorders. In order to evaluate the impact of dopaminergic drugs on outcomes measures, we collected also the levodopa-equivalent dosage at enrolment and at discharge.

\section{Neuropsychological data collection}

In order to understand the relations between neuropsychological aspects and QoL perception, the experimental group was evaluated through a large battery of psychometric tests and scales. These evaluations were performed in the morning, on the third day of hospitalisation, during the medication 'on' state. They included the Mini-Mental State Examination and the Montreal Cognitive Assessment for the global cognitive status; the Frontal Assessment Battery (FAB), the Wisconsin Card Sorting Test (WCST) and the Trail Making Test A and B (TMT A and B) were used to assess the executive functions. Patients were also required to fill in two self-report questionnaires: the Beck Depression Inventory (BDI), aimed to evaluate the presence and the severity of depressive symptoms, and the State-Trait Anxiety Inventory Y (STAI-Y traits and state) in order to define the presence of anxiety disorders.

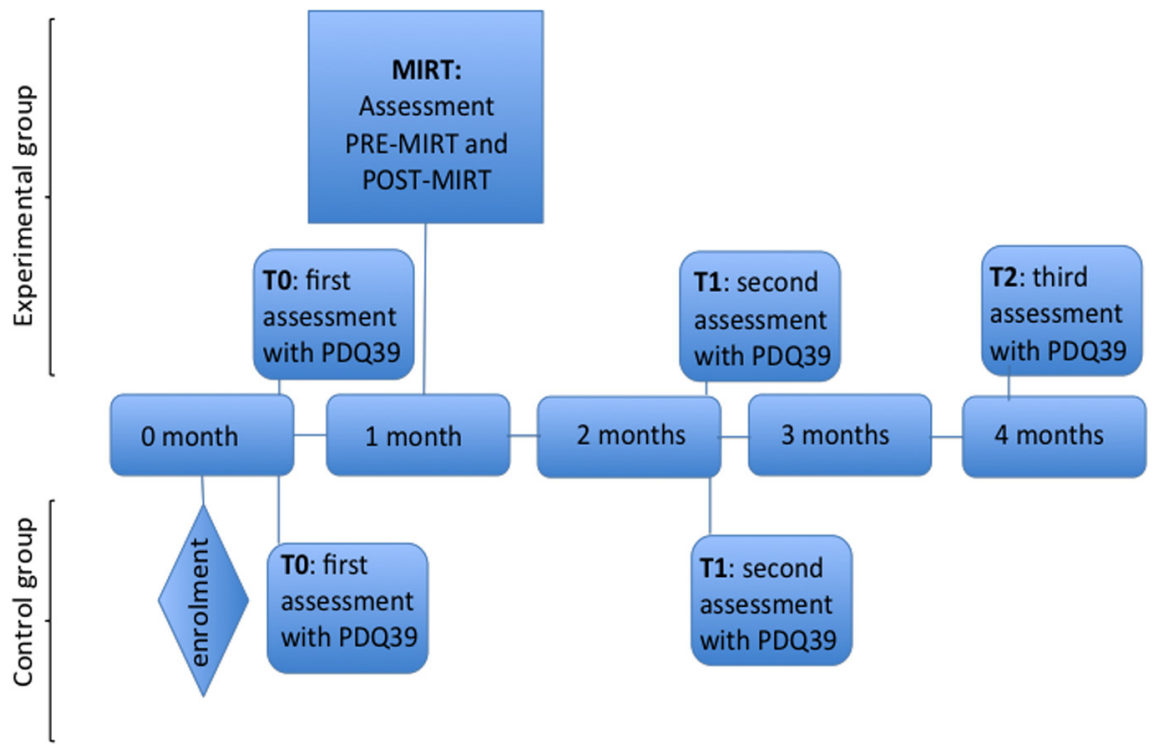

Figure 2 Study design. MIRT, Multidisciplinary Intensive Rehabilitation Treatment; PDQ-39, Parkinson's Disease Questionnaire-39. 
Table 1 Subjects' clinical and demographic data

\begin{tabular}{llll}
\hline Variable & $\begin{array}{l}\text { Controls } \\
(\mathbf{n}=48)\end{array}$ & $\begin{array}{l}\text { Experimental group } \\
(\mathbf{n}=\mathbf{1 8 6 )}\end{array}$ & P value \\
\hline Male (\%) & $30(62.5)$ & $106(57.0)$ & 0.17 \\
Age & $66.9 \pm 10.5$ & $66.5 \pm 8.6$ & 0.77 \\
Hoehn and Yahr stage & $2.6 \pm 0.6$ & $2.6 \pm 0.5$ & 0.75 \\
Disease duration (years) & $7.4 \pm 5.3$ & $9.0 \pm 5.6$ & 0.72 \\
Education (years) & $10.9 \pm 4.2$ & $10.2 \pm 3.9$ & 0.54 \\
\hline
\end{tabular}

\section{Sample size computation}

We computed the sample size according to the two dimensions of PDQ-39 we considered most relevant in our investigation, namely mobility and ADL. The SE of measurement reported in the literature ranges from 6.25 for mobility to 8.54 for ADL (http://www.rehabmeasures.org). We wanted to detect changes at least $50 \%$ greater than the minimally clinically important difference according to Peto and colleagues, ${ }^{35}$ resulting in 4.8 and 6.6 for mobility and ADL, respectively. To detect this change with a two-tailed type I error of 0.05 and a power of $80 \%$, the estimated sample size (the largest between the two estimates) was 170 patients (given the 4:1 allocation ratio, as detailed before, 136 in group 1 and 34 in group 2). The final conservative choice was 250 patients to be randomised (200 in group 1 and 50 in group 2).

\section{Statistical analysis}

Descriptive statistics are given as mean \pm SD. The Shapiro-Wilk statistic was used to test the normality of the distribution of all variables. Primary and secondary outcome measures were non-normally distributed. Accordingly, between-group and within-group comparisons were performed by the Mann-Whitney U test and Wilcoxon signed-rank test, respectively. Comparisons of categorical variables were carried out with the $\mathrm{X}^{2}$ test or Fisher's exact test when appropriate.

To assess the effect of MIRT on PDQ-39 dimensions and on Global Index Score, we compared T1 versus T0 scores within subjects in both experimental and control groups. We also compared the scores between subjects at T0 and at T1. Finally, we computed the delta (within subject, T1-T0) of PDQ-39 and compared the values between subjects.

To investigate whether the improvements in QoL were maintained over a 3-month follow-up period, we compared T2 and T0 PDQ-39 values in the experimental group.
The association between variables was assessed by Spearman's rank correlation coefficient.

The effects of MIRT on the clinical, motor and functional outcomes and on levodopa-equivalent dosage were also assessed by comparing values at $\mathrm{T} 0$ versus $\mathrm{T} 1$.

A P value $<0.05$ was considered statistically significant. When appropriate, false discovery rate was controlled at $5 \%$ using the Benjamini-Hochberg method. A per-protocol analysis was carried out. All analyses were carried out using the SAS/STAT statistical package, release V.9.2.

\section{RESULTS}

We enrolled 250 patients: 200 in the experimental group and 50 in the control group. In the experimental group, eight patients discontinued MIRT for medical complications, two dropped out at T1 and four at T2. Two patients in the control group dropped out. Of the eight patients who discontinued MIRT, three did it for a severe urinary tract infection and five because of a newly diagnosed medical condition: cancer (2), osteoporotic vertebral fracture (1) and heart disease (2). Among the six patients who dropped out at follow-up, three communicated their refusal to continue the study and three did not attend the follow-up interviews.

Consequently, the study population consisted of 186 patients in the experimental group and 48 in the control group (figure 1). In table 1 we report the clinical and demographic data for both groups. No significant differences were observed between the two groups.

In table 2 (left), we report the PDQ-39 scores (all dimensions and Global Index Score) at T0 and T1 for both groups. At T0, no differences were observed between the two groups ( $\mathrm{P}$ value ranging from 0.11 for 'Pain' to 0.90 for 'Social Support'). Comparing PDQ-39 values at T1 versus T0 in the experimental group ( $\mathrm{P}$ values reported in column 7), all dimensions except 'Stigma' significantly improved (all $\mathrm{P}<0.0001$, also after Benjamini-Hochberg adjustment). Contrarily, no significant change was observed in any of the PDQ-39 dimensions and index score in control patients (P values reported in column 4).

In table 2 (right), the PDQ-39 delta values (T1-T0) for both groups are reported. Comparing the deltas between experimental and control group, all dimensions except 'Stigma' were significantly different, even after Benjamini-Hochberg adjustment.

Finally, comparing PDQ-39 values of experimental group at enrolment and after 4 months (T2 vs T0), the Global Index Score maintained a significant improvement, as well as the

Table 2 (Left) PDQ-39 scores (all dimensions and index score) at T0 and T1 for controls and experimental group, and (right) PDQ-39 delta values (T1-T0) for controls and experimental group

\begin{tabular}{|c|c|c|c|c|c|c|c|c|c|}
\hline & \multicolumn{3}{|c|}{ Controls } & \multicolumn{3}{|c|}{ Experimental group } & \multirow{2}{*}{$\begin{array}{l}\text { Controls } \\
\text { Delta T1-T0 }\end{array}$} & \multirow{2}{*}{$\begin{array}{l}\begin{array}{l}\text { Experimental } \\
\text { group }\end{array} \\
\text { Delta T1-T0 }\end{array}$} & \multirow[b]{2}{*}{$\mathbf{P}$} \\
\hline & TO & $\mathrm{T} 1$ & $\mathbf{P}$ & TO & T1 & $\mathbf{P}$ & & & \\
\hline PDQ-39 index score & $41.6 \pm 22.9$ & $42.8 \pm 22.9$ & 0.23 & $43.6 \pm 21.4$ & $35.3 \pm 22.1$ & $<0.0001$ & $1.2 \pm 9.9$ & $-8.3 \pm 18.0$ & $<0.0001$ \\
\hline Mobility & $13.3 \pm 10.7$ & $13.4 \pm 10.4$ & 0.54 & $13.6 \pm 9.3$ & $11.5 \pm 10.2$ & $<0.0001$ & $0.1 \pm 4.8$ & $-2.0 \pm 7.6$ & 0.0047 \\
\hline$A D L$ & $6.8 \pm 5.5$ & $6.7 \pm 5.7$ & 0.36 & $6.7 \pm 5.0$ & $5.6 \pm 5.3$ & $<0.0001$ & $-0.1 \pm 2.9$ & $-1.2 \pm 4.3$ & 0.0160 \\
\hline Emotional well-being & $6.5 \pm 5.0$ & $7.1 \pm 4.7$ & 0.10 & $7.0 \pm 4.6$ & $5.6 \pm 4.7$ & $<0.0001$ & $0.6 \pm 2.8$ & $-1.4 \pm 4.1$ & $<0.0001$ \\
\hline Cognition & $3.3 \pm 2.4$ & $3.4 \pm 2.2$ & 0.49 & $3.8 \pm 2.9$ & $2.5 \pm 2.4$ & $<0.0001$ & $0.1 \pm 1.4$ & $-1.2 \pm 2.5$ & $<0.0001$ \\
\hline Communication & $2.1 \pm 2.4$ & $2.0 \pm 2.1$ & 0.56 & $2.2 \pm 2.2$ & $1.4 \pm 1.7$ & $<0.0001$ & $-0.1 \pm 1.3$ & $-0.8 \pm 2.0$ & 0.0015 \\
\hline Bodily discomfort & $3.1 \pm 2.6$ & $3.2 \pm 2.5$ & 0.65 & $3.7 \pm 2.7$ & $2.9 \pm 2.5$ & $<0.0001$ & $0.1 \pm 1.5$ & $-0.8 \pm 2.4$ & 0.0016 \\
\hline
\end{tabular}

ADL, activities of daily living; PDQ-39, Parkinson's Disease Questionnaire-39. 
Table 3 PDQ-39 values of the experimental group at enrolment and after 4 months (T2 vs T0)

\begin{tabular}{lccc}
\hline Variable & T2 & Delta T2-T0 & P value \\
\hline PDQ-39 index score & $38.8 \pm 20.9$ & $-4.8 \pm 17.5$ & $<0.0001$ \\
\hline Mobility & $13.3 \pm 10.0$ & $-0.2 \pm 7.4$ & 0.48 \\
\hline ADL & $6.0 \pm 5.2$ & $-0.7 \pm 4.3$ & 0.012 \\
\hline Emotional well-being & $5.7 \pm 4.4$ & $-1.3 \pm 3.9$ & $<0.0001$ \\
\hline Stigma & $4.6 \pm 2.2$ & $0.1 \pm 2.7$ & 0.21 \\
\hline Social support & $1.5 \pm 1.9$ & $-0.5 \pm 2.3$ & 0.44 \\
\hline Cognition & $2.9 \pm 2.6$ & $-0.9 \pm 2.5$ & $<0.0001$ \\
\hline Communication & $1.6 \pm 1.9$ & $-0.6 \pm 2.1$ & 0.0020 \\
\hline Bodily discomfort & $3.2 \pm 2.7$ & $-0.5 \pm 2.5$ & 0.0093 \\
\hline ADL activities of daily living: PDQ-39. Parkinson's Disease Questionnaire-39.
\end{tabular}

ADL, activities of daily living; PDQ-39, Parkinson's Disease Questionnaire-39.

'ADL', 'Well-Being', 'Cognition', 'Communication' and 'Pain' dimensions (all $\mathrm{P}<0.02$ after Benjamini-Hochberg adjustment) (table 3). The 'Mobility', 'Stigma' and 'Social Support' dimensions returned to values not significantly different from baseline.

In table 4 we report the clinical, motor and functional outcomes: comparing pre-MIRT versus post-MIRT, total UPDRS, TUG, PDDS and BBS improved significantly (all $\mathrm{P}<0.0001$ ).

Levodopa-equivalent dosage significantly decreased after MIRT (from $583 \pm 327$ to $528 \pm 300 \mathrm{mgeq} / \mathrm{die}, \mathrm{P}<0.0001$ ).

The correlation analysis showed at T0 a significant association between levodopa-equivalent dosage and PDQ-39 Global Index Score $(P=0.02$, with Spearman's $r=0.18)$. At $T 1$ the same association was non-significant $(r=0.11, \mathrm{P}=0.13)$.

Assessing the relationship between QoL at T0 and clinical, motor and functional variables pre-MIRT, we found a significant association between PDQ-39 index score and total UPDRS, TUG, PDDS and BBS (Spearman's r: 0.51, 0.41, 0.44 and -0.43 , respectively, all $\mathrm{P}<0.0001)$. These associations were also significant at discharge (Spearman's r: 0.41, 0.38, 0.42, -0.32, respectively, all $\mathrm{P}<0.0001$ ).

The PDQ-39 index score at T0 was significantly associated with BDI $(r=0.53, P<0.0001)$ and STAI-Y trait $(r=0.34$, $\mathrm{P}<0.0001)$, but not associated with STAI-Y state $(\mathrm{r}=-0.05$, $\mathrm{P}=0.48)$.

No association was found at T0 between the PDQ-39 index score and FAB, TMT A, TMT B, TMT B-A, WCST and Stroop test time scores (all $\mathrm{P}>0.15)$. A weak but significant association was found with Stroop test errors score $(r=0.16, P=0.04)$.

\section{DISCUSSION}

The main finding of this study is that a multidisciplinary, aerobic, intensive and motor-cognitive rehabilitation treatment such as MIRT, properly tailored for PD, might induce benefits on patients' self-perceived QoL. Remarkably, we observed that this improvement was maintained along a 3-month follow-up period.

Table 4 Clinical, motor and functional outcomes: comparing preMIRT versus post-MIRT

\begin{tabular}{lllll}
\hline Variable & Pre-MIRT & Post-MIRT & \multicolumn{1}{l}{$\begin{array}{l}\text { Delta pre-post } \\
\text { MIRT }\end{array}$} & P value \\
\hline Total UPDRS & $39.6 \pm 10.1$ & $27.2 \pm 9.3$ & $-12.4 \pm 4.1$ & $<0.0001$ \\
BBS & $46.8 \pm 8.7$ & $52.5 \pm 6.3$ & $5.7 \pm 4.4$ & $<0.0001$ \\
TUG & $13.3 \pm 9.8$ & $10.4 \pm 8.7$ & $-2.9 \pm 8.2$ & $<0.0001$ \\
PDDS & $75.7 \pm 12.6$ & $58.6 \pm 12.1$ & $-17.1 \pm 8.1$ & $<0.0001$ \\
\hline
\end{tabular}

BBS, Berg Balance Scale; MIRT, motor-cognitive and intensive rehabilitation treatment; PDDS, Parkinson's Disease Disability Scale; TUG, Timed Up and Go Test; UPDRS, Unified Parkinson's Disease Rating Scale.
These findings strengthen the evidence about the efficacy of a multidisciplinary rehabilitation treatment on patients with PD. ${ }^{11} 12242530$ In order to evaluate the impact of the treatment on the different areas of QoL, investigated by PDQ-39, we analysed both the Global Index Score and each subscore of the questionnaire. Our results showed that all dimensions improved in the month following the treatment (T1 vs T0), with the exception of 'stigma'. This finding means that patients' self-perception of mobility, autonomy in ADLs, emotional well-being, needing of social support, cognition, communicative abilities and bodily discomfort are enhanced after MIRT.

The invariance in self-perception of 'stigma' is worthy of discussion. People with PD may experience stigma, such as shame, embarrassment and disgrace. Sociocultural context is considered the major determinant of stigma, ${ }^{36}$ which in turn is an important aspect of QoL in people with PD. Moreover, stigma is associated with depressive symptoms and motor difficulties. ${ }^{36}$ According to these concepts, we found a direct association between depressive and anxiety symptoms and QoL at enrolment. At the same evaluation, there was also a positive correlation between 'stigma' subdomain score and all other PDQ-39 subdomains. Interestingly, this correlation was lost at follow-up. We argue that the factor "stigma", even though unresponsive to the treatment, does not affect the effectiveness of MIRT on QoL.

When evaluating the possible impact of cognitive performances at baseline on QoL, we did not find any significant association between the cognitive tests (FAB, TMT A, TMT B, TMT B-A, WCST) and PDQ-39, with the exception of Stroop test, which evaluates an executive component of attention. This suggests that QoL is not directly associated with other executive functions, but is reduced in those patients with lower capacity of interference suppression. This aspect is relevant because it suggests a direct connection between exercise, executive functions and QoL. The beneficial effect of physical therapies and aerobic training in improving cognitive functions and learning, ${ }^{37-39}$ as well as QoL, ${ }^{40}$ has been already highlighted in previous studies. More specifically, Lawson $e t a l^{41}$ showed that interventions able to improve attention could potentially improve QoL. Consistent with this evidence, we have have demonstrated that an intensive and aerobic rehabilitation treatment such as MIRT promotes a beneficial effect on the executive component of attention in patients with PD. ${ }^{42}$

At the 3-month follow-up, despite the self-perception of motor abilities ('mobility' subitem of PDQ-39) coming back to baseline score, patients maintained a better perception of QoL, demonstrated by the maintenance of the achieved improvement of the Global Index and the other subscale scores.

Considering the secondary outcomes, MIRT induced significant improvements in the clinical, motor and functional measures, in accordance with previous findings from our group. ${ }^{1112}$ Another interesting finding concerns the correlation between PDQ-39 score and the amount of dopaminergic therapy.

During the rehabilitative hospitalisation, with the improvement of patients' motor performances, it is indeed possible to reduce the dosage of the dopaminergic therapy, in order to treat drug-related dyskinesias. ${ }^{11} 1243$ However, the consequent reduction of dyskinesias cannot be totally attributed to the decrease in the total amount of dopaminergic drugs. The cellular basis of dyskinesias relies in the loss of the synaptic depotentiation at the corticostriatal level. ${ }^{44}$ Exaggerated movements in response to the stimulation of dopaminergic receptors, such as those occurring during dyskinesias, might lead erroneous information to the motor striatal circuits. Therefore, when concomitant and competing correct movements are performed, as during 
rehabilitation treatment, the manifestation of abnormal dyskinetic movements may be attenuated. ${ }^{43456}$

While at baseline the finding of a positive correlation between levodopa-equivalent dosage and PDQ-39 index score indicates a direct effect of dopaminergic therapy on self-perceived QoL, the lost of this correlation at the end of treatment suggests that the overall improvement in QoL is related to the effect of MIRT itself and not to the pharmacological treatment.

Therefore, it is arguable that the improvement in patients' perception of QoL was related to the rehabilitative treatment rather than to the dopaminergic therapy. These observations confirm the relevance of a synergism between medical therapies and exercise able to improve QoL of patients with PD. ${ }^{11} 1247$ Indeed, exercise and rehabilitation, improving motor performances and autonomy in the activities of daily life, reduce the need to increase dopaminergic therapy ${ }^{11} 12$ and decrease the risk of drug-related side effects impacting on QoL. ${ }^{67}$

\section{Study limitations}

There are some limitations to this study that have to be acknowledged. First, we evaluated QoL over a 3-month follow-up period. This allows us to affirm that MIRT acts positively on QoL, but a longer follow-up would be useful to investigate the persistence of beneficial effects over time. Second, we did not assess UPDRS, PDDS, TUG and BBS at follow-up in the experimental group. In the absence of these data, we cannot determine whether the benefit from the treatment was maintained even in these parameters, and to what extent they affected QoL in the long-term period.

The single-blind design of this study could be another limitation due to the possible placebo effect. This problem has to be addressed considering that the rehabilitative context makes it impossible to obtain a double-blinded study design. Moreover, MIRT has been tested in previous studies, and its effectiveness on motor, clinical and functional outcomes has been found to be closely related with objective metabolic measures ${ }^{48}$ and with changes in the plasmatic levels of biochemical and molecular markers of neuroplasticity (such as brain-derived neurotrophic factor and brain-derived neurotrophic factorTrkB). ${ }^{19} 49$ Since the improvement in PDQ-39 Global Index Score was maintained after a 3-month follow-up period, we think that the hypothesis of a placebo effect on outcomes improvement is unlikely. Nevertheless, the lack of blinding of the neurologist and the physiotherapist who performed the assessments and the unsolved question about how to improve the design of the control group in order to reduce the placebo effect certainly are limitations of this study that have to be acknowledged. In further studies, a possible solution could be to enrol a control group of patients who undergo a 4-week rehabilitative treatment with the same intensity of MIRT but without specificity or vice versa.

Finally, we did not use specific outcome measures for speech, swallowing and dexterity despite the evidence that the intervention is a result of the total package, with presumed outcomes well beyond mobility. This should be considered as a study limitation. Nevertheless, among the secondary outcomes, we chose the total UPDRS, which allows evaluation of the motor performance and different ADLs such as eating, dressing, writing, talking and the dexterity. We assessed the scale both at hospital admission and at discharge. We have shown that the total UPDRS significantly improved at the end of MIRT, as a result of an improvement in all the different aspects of the disease. These data confirm the results from our previous studies. ${ }^{11} 12$ Also the PDDS, a specific self-administered scale for evaluating ADLs, was used as secondary outcome and it improved significantly after the MIRT. Moreover, the 6 minute walk test (6MWT), BBS and TUG scores highlight the benefits achieved on gait and balance disturbances, which have been recognised as the most impactful on the QoL of patients with PD. ${ }^{50}$ Overall, these data confirm the effectiveness of MIRT on motor symptoms, as well as its effectiveness on ADLs and speech, thus reflecting the effects of the occupational therapy and of the speech therapy performed during the hospitalisation. Certainly, future studies with more appropriate outcome measures to assess specific aspects of the disease, such as the speech and ADLs, should be designed.

Further studies are needed to clarify these issues and to better understand the impact of tailored rehabilitation programmes on QoL of patients suffering from PD.

\section{Open questions and future directions}

In this study, we have shown that a rehabilitation programme such as MIRT improves QoL of people living with PD and that the overall effect is maintained after a 3-month follow-up period. These results are very impactful for patients as well as clinicians.

However, data from this study raise several questions about the sustainability of this programme in terms of costs and patients' clinical management.

The question of cost:benefit ratio is strictly dependent on the different national healthcare systems. It is known that PD has an enormous social and medical economic impact ${ }^{51}$ and that patients with PD can take advantages from pharmacological and surgical therapies whose effectiveness have already been demonstrated. However, those treatments are expensive as well $^{52}$ and, in a relevant percentage of cases, the pharmacological therapy imposes a significant economic burden in terms of related side effects. ${ }^{53}$ Therefore, the costs of a treatment such as MIRT seem to be rewarded by its effectiveness in the short-term and long-term period. ${ }^{11}{ }^{12}$ Furthermore, as it has been shown, rehabilitation allows a reduction or a non-increase in the total amount of dopaminergic drugs, thus probably compensating the costs of the global management of PD. ${ }^{1112}$

Other crucial questions concern the daily management of patients after the end of treatment. At discharge, our group consigns to each patient a programme of tailored home-based exercises. Nevertheless, we do not follow the patients' adherence to this programme: for this reason, our next step will be to create a project for the implementation of the patients' care and follow-up at home, in order to favour the maintenance of the improvement in motor, functional and speech performances. For example, by adopting the ParkinsonNet approach used in the Netherlands, ${ }^{54}$ a speech and language therapist or physiotherapist working in the community close to the living environment of the patient or perhaps a PD nurse could be considered. After MIRT these healthcare professionals could play an important role in stimulating and motivating patients and maintaining the treatment effects. Other possible strategies aimed to improve patients' compliance to the 'home-based' therapies may include the use of specific electronic devices, such as smartphone applications or web trainings.

Finally, a possible direction for the future could be the possibility to hospitalise patients every time after a significant worsening of their motor condition, in spite of an optimal management of the pharmacological or surgical therapies, to undergo a 'boost rehabilitative intervention'. 
Contributors DF: wrote the text, conceived and designed the experiments, provided substantial contributions to discussion and edited the manuscript before submission. PO: wrote the text, conceived and designed the experiments, performed the experiments, provided substantial contributions to discussion and generated the figures. IZ: wrote the text, conceived and designed the experiments, and provided substantial contributions to discussion. VC: researched data for the article. EU: researched data for the article. MFG: provided substantial contributions to discussion. RM: analysed the data and did the statistical analysis. GF: conceived and designed the experiments, analysed the data, wrote the text and provided substantial contributions to discussion.

\section{Competing interests None declared.}

Patient consent Obtained.

Ethics approval The study design and protocol were approved by the local scientific committee ('Moriggia-Pelascini' Hospital, Gravedona e Uniti - Como) and were in accordance with the Code of Ethics of the World Medical Association (Declaration of Helsinki, 1967).

Provenance and peer review Not commissioned; externally peer reviewed.

Open access This is an open access article distributed in accordance with the Creative Commons Attribution Non Commercial (CC BY-NC 4.0) license, which permits others to distribute, remix, adapt, build upon this work non-commercially, and license their derivative works on different terms, provided the original work is properly cited and the use is non-commercial. See: http://creativecommons.org/ licenses/by-nc/4.0/

(C) Article author(s) (or their employer(s) unless otherwise stated in the text of the article) 2018. All rights reserved. No commercial use is permitted unless otherwise expressly granted.

\section{REFERENCES}

1 Dowding $\mathrm{CH}$, Shenton $\mathrm{CL}$, Salek SS. A review of the health-related quality of life and economic impact of Parkinson's disease. Drugs Aging 2006;23:693-721.

2 Ebrahim S. Clinical and public health perspectives and applications of health-related quality of life measurement. Soc Sci Med 1995;41:1383-94

3 Chaudhuri KR, Healy DG, Schapira AH. National Institute for Clinical Excellence. Nonmotor symptoms of Parkinson's disease: diagnosis and management. Lancet Neurol $2006: 5: 235-45$

4 Martinez-Martin P, Rodriguez-Blazquez C, Forjaz MJ, et al. Impact of pharmacotherapy on quality of life in patients with Parkinson's Disease. CNS Drugs 2015;29:397-413.

5 Gallagher DA, Schrag A. Impact of newer pharmacological treatments on quality of life in patients with Parkinson's disease. CNS Drugs 2008:22:563-86.

6 Behari M, Srivastava AK, Pandey RM. Quality of life in patients with Parkinson's disease. Parkinsonism Relat Disord 2005:11:221-6.

7 Chapuis S, Ouchchane L, Metz O, et al. Impact of the motor complications of Parkinson's disease on the quality of life. Mov Disord 2005;20:224-30.

8 Tomlinson CL, Patel S, Meek C, et al. Physiotherapy versus placebo or no intervention in Parkinson's disease. Cochrane Database Syst Rev 2012;7:CD002817.

9 Bloem BR, de Vries NM, Ebersbach G. Nonpharmacological treatments for patients with Parkinson's disease. Mov Disord 2015:30:1504-20.

10 Goodwin VA, Richards SH, Taylor RS, et al. The effectiveness of exercise interventions for people with Parkinson's disease: a systematic review and meta-analysis. Mov Disord 2008;23:631-40.

11 Frazzitta G, Bertotti G, Riboldazzi G, et al. Effectiveness of intensive inpatient rehabilitation treatment on disease progression in parkinsonian patients: a randomized controlled trial with 1-year follow-up. Neurorehabil Neural Repair 2012;26:144-50.

12 Frazzitta G, Maestri $R$, Bertotti $G$, et al. Intensive rehabilitation treatment in early Parkinson's disease: a randomized pilot study with a 2-year follow-up. Neurorehabil Neural Repair 2015;29:123-31.

13 Ekker MS, Janssen S, Nonnekes J, et al. Neurorehabilitation for Parkinson's disease: Future perspectives for behavioural adaptation. Parkinsonism Relat Disord 2016;22:S73-7

14 Canning CG, Sherrington C, Lord SR, et al. Exercise for falls prevention in Parkinson disease: a randomized controlled trial. Neurology 2015;84:304-12.

15 Smania N, Corato E, Tinazzi M, et al. Effect of balance training on postural instability in patients with idiopathic Parkinson's disease. Neurorehabil Neural Repair 2010;24:826-34.

16 Nieuwboer A, Kwakkel G, Rochester L, et al. Cueing training in the home improves gait-related mobility in Parkinson's disease: the RESCUE trial. J Neurol Neurosurg Psychiatry 2007:78:134-40.

17 Hirsch MA, lyer SS, Sanjak M. Exercise-induced neuroplasticity in human Parkinson's disease: What is the evidence telling us? Parkinsonism Relat Disord 2016;22:S78-81.

18 Petzinger GM, Fisher BE, McEwen S, et al. Exercise-enhanced neuroplasticity targeting motor and cognitive circuitry in Parkinson's disease. Lancet Neurol 2013;12:716-26.
19 Fontanesi C, Kvint S, Frazzitta G, et al. Intensive rehabilitation enhances lymphocyte BDNF-TrkB signaling in patients with Parkinson's Disease. Neurorehabil Neural Repair 2016;30:411-8.

20 Herman T, Giladi N, Gruendlinger $\mathrm{L}$, et al. Six weeks of intensive treadmill training improves gait and quality of life in patients with Parkinson's disease: a pilot study. Arch Phys Med Rehabil 2007:88:1154-8.

21 Paker N, Bugdayci D, Goksenoglu G, et al. Effects of robotic treadmill training on functional mobility, walking capacity, motor symptoms and quality of life in ambulatory patients with Parkinson's disease: a preliminary prospective longitudina study. NeuroRehabilitation 2013;33:323-8.

22 Morberg BM, Jensen J, Bode $M$, et al. The impact of high intensity physical training on motor and non-motor symptoms in patients with Parkinson's disease (PIP): a preliminary study. NeuroRehabilitation 2014;35:291-8.

23 Peto $V$, Jenkinson C, Fitzpatrick $R$, et al. The development and validation of a short measure of functioning and well being for individuals with Parkinson's disease. Qual Life Res 1995;4:241-8.

24 Giladi N, Manor Y, Hilel A, et al. Interdisciplinary teamwork for the treatment of people with Parkinson's disease and their families. Curr Neurol Neurosci Rep 2014;14:493.

25 Post B, van der Eijk M, Munneke M, et al. Multidisciplinary care for Parkinson's disease: not if, but how!. Postgrad Med J 2011;87:575-8.

26 Rochester L, Espay AJ. Multidisciplinary rehabilitation in Parkinson's disease: a milestone with future challenges. Mov Disord 2015;30:1011-3.

27 Tan SB, Williams AF, Kelly D. Effectiveness of multidisciplinary interventions to improve the quality of life for people with Parkinson's disease: a systematic review. Int I Nurs Stud 2014;51:166-74.

28 van der Marck MA, Munneke M, Mulleners W, et al. Integrated multidisciplinary care in Parkinson's disease: a non-randomised, controlled trial (IMPACT). Lancet Neurol 2013;12:947-56.

29 van der Marck MA, Bloem BR, Borm GF, et al. Effectiveness of multidisciplinary care for Parkinson's disease: a randomized, controlled trial Mov Disord 2013:28:605-11.

30 Frazzitta G, Bertotti G, Uccellini D, et al. Parkinson's disease rehabilitation: a pilot study with 1 year follow up. Mov Disord 2010;25:1762-3.

31 Hughes AJ, Daniel SE, Kilford L, et al. Accuracy of clinical diagnosis of idiopathic Parkinson's disease: a clinico-pathological study of 100 cases. J Neurol Neurosurg Psychiatry 1992:55:181-4.

32 Frazzitta G, Maestri R, Uccellini D, et al. Rehabilitation treatment of gait in patients with Parkinson's disease with freezing: a comparison between two physical therapy protocols using visual and auditory cues with or without treadmill training. Mov Disord 2009:24:1139-43.

33 Frazzitta G, Bossio F, Maestri R, et al. Crossover versus stabilometric platform for the treatment of balance dysfunction in Parkinson's Disease: a Randomized Study. Biomed Res Int 2015;2015.

34 Opara JA, Brola W, Leonardi M, et al. Quality of life in Parkinson's disease. J Med Life 2012:5:375-81.

35 Peto V, Jenkinson C, Fitzpatrick R. Determining minimally important differences for the PDQ-39 Parkinson's disease questionnaire. Age Ageing 2001;30:299-302.

$36 \mathrm{Ma} \mathrm{HI}$, Saint-Hilaire M, Thomas CA, et al. Stigma as a key determinant of healthrelated quality of life in Parkinson's disease. Qual Life Res 2016:25:3037-45.

37 David FJ, Robichaud JA, Leurgans SE, et al. Exercise improves cognition in Parkinson's disease: The PRET-PD randomized, clinical trial. Mov Disord 2015;30:1657-63.

38 Duchesne C, Lungu O, Nadeau A, et al. Enhancing both motor and cognitive functioning in Parkinson's disease: aerobic exercise as a rehabilitative intervention. Brain Cogn 2015;99:68-77.

39 Manenti R, Brambilla M, Benussi A, et al. Mild cognitive impairment in Parkinson's disease is improved by transcranial direct current stimulation combined with physical therapy. Mov Disord 2016;31:715-24.

40 Uc EY, Doerschug KC, Magnotta V, et al. Phase I/II randomized trial of aerobic exercise in Parkinson disease in a community setting. Neurology 2014;83:413-25.

41 Lawson RA, Yarnall AJ, Duncan GW, et al. Cognitive decline and quality of life in incident Parkinson's disease: The role of attention. Parkinsonism Relat Disord 2016;27:47-53.

42 Ferrazzoli D, Ortelli P, Maestri R, et al. Focused and sustained attention is modified by a goal-based rehabilitation in Parkinsonian Patients. Front Behav Neurosci 2017;11:56.

43 Frazzitta G, Bertotti G, Morelli M, et al. Rehabilitation improves dyskinesias in Parkinsonian patients: a pilot study comparing two different rehabilitative treatments. NeuroRehabilitation 2012;30:295-301.

44 Picconi B, Centonze D, Håkansson K, et al. Loss of bidirectional striatal synaptic plasticity in L-DOPA-induced dyskinesia. Nat Neurosci 2003;6:501-6.

45 Simola N, Morelli M, Frazzitta G, et al. Role of movement in long-term basal ganglia changes: implications for abnormal motor responses. Front Comput Neurosci 2013:7:142.

46 Frazzitta G, Morelli M, Bertotti G, et al. Intensive rehabilitation treatment in parkinsonian patients with dyskinesias: a preliminary study with 6-month followup. Parkinsons Dis 2012:2012:1-4.

47 Ferrazzoli D, Carter A, Ustun FS, et al. Dopamine replacement therapy, learning and reward prediction in Parkinson's Disease: implications for rehabilitation. Front Behav Neurosci 2016;10:121. 
48 Frazzitta G, Maestri R, Bertotti G, et al. Rehabilitation in Parkinson's disease: assessing the outcome using objective metabolic measurements. Mov Disord 2010;25:609-14.

49 Frazzitta G, Maestri R, Ghilardi MF, et al. Intensive rehabilitation increases BDNF serum levels in parkinsonian patients: a randomized study. Neurorehabil Neural Repair 2014;28:163-8.

50 Ortelli P, Maestri R, Zarucchi M, et al. Italian validation of the Belastungsfragebogen Parkinson kurzversion (BELA-P-k): a disease-specific questionnaire for evaluation of the subjective perception of quality of life in parkinson's disease. J Clin Mov Disord 2017:4:12.
51 Dodel RC, Singer M, Köhne-Volland R, et al. The economic impact of Parkinson's disease. An estimation based on a 3-month prospective analysis. Pharmacoeconomics 1998;14:299-312.

52 Dodel RC, Eggert KM, Singer MS, et al. Costs of drug treatment in Parkinson's disease. Mov Disord 1998;13:249-54.

53 Suh DC, Pahwa R, Mallya U. Treatment patterns and associated costs with Parkinson's disease levodopa induced dyskinesia. J Neuro/ Sci 2012;319:24-31.

54 Bloem BR, Munneke M. Revolutionising management of chronic disease: the Parkinson Net approach. BMJ 2014;348:g1838. 